FORMATION Formation emploi

Revue française de sciences sociales

$110 \mid 2010$

Pêle-mêle

\title{
La nouvelle condition des étudiants anglais : entre endettement et emploi
}

The new state of students in england: between debt and employment

Neue verhältnisse für britische studenten: zwischen verschuldung

underwerbstätigkeit

La nueva condición de los estudiantes ingleses: entre endeudamiento y empleo

\section{Aurélien Casta}

\section{(Q) OpenEdition}

Journals

Édition électronique

URL : http://journals.openedition.org/formationemploi/3007

DOI : 10.4000/formationemploi.3007

ISSN : 2107-0946

Éditeur

La Documentation française

Édition imprimée

Date de publication : 15 juin 2010

Pagination : 19-34

ISSN : 0759-6340

Référence électronique

Aurélien Casta, "La nouvelle condition des étudiants anglais : entre endettement et emploi », Formation emploi [En ligne], 110 | 2010, mis en ligne le 10 septembre 2012, consulté le 30 octobre 2020. URL : http://journals.openedition.org/formationemploi/3007 ; DOI : https://doi.org/10.4000/ formationemploi.3007 


\title{
La nouvelle condition des étudiants anglais: entre endettement et emploi ${ }^{1}$
}

\author{
Par Aurélien Casta*
}

\section{Suite aux politiques publiques d'enseignement supérieur menées au Royaume-Uni, les effectifs ont doublé depuis 1987. Cela s'est accompagné d'une augmentation des frais d'inscription et des prêts étudiants qui a généré l'endettement et la mise en emploi des étudiants.}

Les frais d'inscription et l'endettement étudiant initiés, de 1990 à 2006, par la politique britannique dans le cadre de l'expansion de l'enseignement supérieur, modifient-ils les réalités sociales étudiantes du pays? Nous confronterons ici les effets de cette politique aux objectifs qui lui étaient initialement assignés.

Ces interrogations se justifient tout d'abord pour des raisons d'ordre théorique. Il nous semble en effet que l'objet a suscité très peu de travaux sociologiques francophones, annihilant par la même occasion toute perspective de comparaison internationale sur les questions étudiantes ${ }^{2}$. Dans le champ des sciences sociales, les économistes se sont déjà saisis de cet

${ }^{1}$ Cet article doit beaucoup au Centre for Employment Research (CER) de la London Westminster University et à Linda Clarke, qui ont accueilli l'auteur au premier semestre de l'année 2009, à l'occasion d'un séjour de recherche. Qu'ils en soient ici remerciés. 2 À titre d'exemple, le livre de synthèse d'Olivier Galland et Marco Oberti (1996) sur les étudiants traite de leurs revenus mais ne s'appuie, comme dans l'ensemble du livre, que sur des travaux relatifs au cas français. Les enquêtes de l'Observatoire de la vie étudiante posent le même problème. Les travaux d'Eric Maurin (2007) s'attachant à montrer le lien entre frais d'inscription, prêts objet pour l'intégrer en général aux problématiques de l'analyse économique standard ${ }^{3}$, centrées notamment sur l'incapacité des mécanismes de marché à assurer une coordination optimale des décisions individuelles concernant les biens publics comme l'enseignement

étudiants et expansion de l'enseignement supérieur en Australie font ici figure d'exception.

${ }^{3}$ L'intervention de Robert Gary-Bobo et d'Alain Trannoy (2005), en faveur de la mise en place de frais d'inscription et de prêts étudiants en France, et la recherche collective de l'Institut de recherche sur l'éducation (IREDU) (1977) sur l'aide aux étudiants en France illustrent bien l'intégration de notre objet au cadre d'analyse économique standard. Plus original, l'article d'Annie Vinokur (2007) sur la doctrine du " partage des côuts » et les nouveaux rapports de pouvoir qu'elle organise dans l'enseignement supérieur, présente aussi un grand intérêt.

* Aurélien Casta est doctorant en sociologie à l'université Paris Ouest Nanterre, Institut des dynamiques historiques et économiques ICNRS - UMR [unité mixte de recherche] 8533). Ses thèmes de recherche portent sur les étudiants, les politiques sociales, l'enseignement supérieur. 
supérieur ; source déterminante dans le financement de l'enseignement supérieur, l'État doit, d'une part, « trouver le bon prix » du bien afin de garantir l'efficacité des mécanismes de marché quant à la production et à l'allocation des ressources et, d'autre part, s'assurer, par la distribution de ressources monétaires adéquates et au nom de l'équité, que les individus ont les mêmes capacités économiques d'accès au bien (Eymard-Duvernay et Favereau, 2006). Dans la lignée du programme général de recherche esquissé par Georg Simmel (1987; 1998), il nous semble que les subventions demandées (ou non) et allouées aux étudiants par l'État n'ont pas seulement vocation à assurer des objectifs économiques quantitativement normés (taux de croissance, niveau de salaire, taux d'accès aux études supérieures...). En tant qu'objet monétaire et politique, ces subventions illustrent les relations entre les étudiants et la collectivité et permettent, tout autant qu'elles expriment, la valorisation des études supérieures. Ces subventions constituent un fait social, détaché des sujets producteurs et consommateurs, susceptible de modifier les réalités d'usage et de travail qui lui préexistent (Simmel, op. cit.) au sein de la population étudiante (temps de travail encadré et personnel, nature et niveau des pratiques culturelles et de l'activité rémunérée exercée en parallèle aux études...). Elles constituent donc un objet dont les objectifs politiques et les effets nécessitent une réflexion sociologique.

La recherche se justifie ensuite pour des raisons ayant trait à l'action publique britannique et à sa mobilisation progressive comme référentiel par les décideurs politiques français. Cette mobilisation a connu, récemment, un moment fort et significatif ${ }^{4}$ avec la proposition de loi, enregistrée le 21 janvier 2009 à l'Assemblée nationale ${ }^{5}$, qui instaure un système de prêts étudiants sans conditions d'accès, dont le

\footnotetext{
${ }^{4}$ Les systèmes de prêts antérieurs, avec des budgets publics volontairement limités, ne pouvaient concerner qu'une infime partie de la population étudiante. Le dernier dispositif instauré par Valérie Pécresse, en septembre 2008, pourtant plus ambitieux que les précédents, a lui aussi des moyens limités et n'offre aucune garantie sur les taux d'intérêt.

5 www.assemblee-nationale.fr/13/propositions/pion1391.asp
}

\section{Encadré 1}

\section{Sources et méthodes}

L'article confronte, d'une part, un corpus de textes produits ou commandés par le gouvernement britannique à des fins directement législatives (livres blancs ou rapports); d'autre part, un ensemble de cinq enquêtes statistiques sur les revenus et les dépenses des étudiants, commandées tous les 3 ou 4 ans par le ministère en charge de l'enseignement supérieur, depuis 1988.

Le corpus est limité aux textes traitant directement des politiques publiques en matière d'enseignement supérieur et de ressources monétaires étudiantes. II s'agit ici de déterminer comment s'articulent ces deux objets. Le corpus sélectionné est le suivant :

Department For Education and Science : (1987), Higher education: meeting the challenge.

(1988), Top-up loans for students. (1991), Higher education: a new framework.

Dearing (sous la dir.) (1997), Higher Education in a learning society, National Committee of Inquiry into Higher Education.

Clarke C. (2003), The future of higher education, white paper on higher education, DIES.

Department For Education And Skills (2004), Moving toward a single combined grant for higher education, DIES.

Les études commandées par le gouvernement britannique, de 1988 à 2004, et intitulées " Enquêtes sur les revenus et les dépenses des étudiants " (Student income and expenditure surveys; SIES), permettent d'évaluer la recomposition du revenu moyen des étudiants anglais :

Windle R. (1989), SIES 1988/89, Research Services Limited.

Windle R. (1993), SIES 1992/93, Research Services Limited. 


\section{(Suite Encadré 1)}

Callender C. (1996), SIES 1995/96 Student finances: income, expenditure and take-up of student loans, Policy Studies Institute.

Callender C. et Kemp (2000), SIES 1998/99 Changing student finances: income, expenditure and the take-up of student loans among full- and part-ime higher education students in 1998/99, DIEE.

Cebulla A., Connor H., Finch S., Hillage J., Hunt W., Jones A., Loukas G., Parfrement J., Pollard E. et Tyers C. (2006), SIES 2004/05, DIES.

En regroupant d'un côté les enquêtes de 1989, 1993 et 1996 et celles de 1999 et 2005 de l'autre, il est possible de confronter les évolutions. La méthodologie adoptée par ces grandes enquêtes est variable sous de nombreux aspects. À titre d'exemple, la méthode de mesure du revenu dans les deux dernières enquêtes exclut l'épargne et les crédits commerciaux, et mesure le poids des prestations sociales qui étaient auparavant intégrées dans le poste "autre ». Comme le soulignent les auteurs, cela n'empêche pas les comparaisons; nous avons repris quatre grandes simplifications opérées par les auteurs.

Tout d'abord, les trois premières enquêtes traitent uniquement des étudiants à temps plein et s'efforcent d'expliquer les différences entre étudiants de plus et de moins de 26 ans. Les deux dernières intègrent (tout en les traitant à part) les étudiants à temps partiel. Le cas de ces étudiants sera davantage abordé dans la dernière partie. Les données exposées par la suite à partir des trois premières enquêtes ne concernent que les étudiants de moins de 26 ans, ceux-ci représentant, au moment des enquêtes, plus de $80 \%$ des étudiants à temps plein.

Ensuite, les enquêtes (par questionnaire et par entretien) ont été menées auprès de plusieurs milliers d'étudiants britanniques, mais la dernière enquête ne traite que des étudiants inscrits dans des établissements d'enseignement supérieur situés au Pays de Galles et en Angleterre. Faisant l'hypothèse que la situation financière des étudiants britanniques était homogène sur l'ensemble du territoire britannique avant 1999, nous réduirons, dans la suite de l'article, notre propos aux "étudiants anglais ", à savoir ici des étudiants inscrits dans les établissements d'enseignement supérieur du territoire anglais (131 des 165 établissements britanniques).

Par ailleurs, suivant la ligne tracée par les politiques publiques britanniques et le champ d'éligibilité des ressources étudiées, nous réduisons notre propos aux étudiants inscrits au niveau "undergraduate ", c'est-à-dire inscrits dans les trois premières années du cursus universitaire britannique.

Les effectifs lors de l'année universitaire 2004/05 montrent que la population étudiée dans l'article représente $58 \%$ des étudiants du territoire britannique, et les trois quarts des étudiants au niveau de la licence (HESA, 2006).

Enfin, la quatrième partie de l'article repose sur les catégories socio-économiques britanniques / social class » ou "socio-economic group»). Leur construction consiste à croiser les critères liés à la relation d'emploi (employeur, employé, indépendant...) et les critères liés au poste occupé.

Les classes I et II regroupent ainsi les employeurs et les cadres supérieurs, la classe IIIn les professions intermédiaires non manuelles, la classe IIIm les professions intermédiaires manuelles et les classes IV et $V$ les professions manuelles, ordinaires et faiblement qualifiées ainsi que les personnes sans emploi.

remboursement serait organisé une fois les études terminées, à partir d'un certain niveau de rémunération. La proposition n'a jusqu'ici pas abouti. Les 92 députés signataires estimaient qu'une telle proposition permettrait de combler les défaillances du système fondé sur la gratuité et les bourses, en permettant « d'une part, de faciliter l'accès des jeunes à l'enseignement supérieur [...], d'autre part d'accom- pagner ce financement privé d'une obligation implicite d'efficacité de l'enseignement supérieur ». Si la hausse des frais d'inscription n'est pas directement évoquée, les parlementaires jugent que le système de prêts proposé permettra de mobiliser des « financements privés » ou « complémentaires » à ceux assurés par la collectivité. Les exemples étrangers (Australie, Nouvelle-Zélande...) mobilisés par les signataires de 
la proposition sont révélateurs de leur volonté d'associer un tel système de prêts au développement des frais d'inscription. Le Royaume-Uni, qui aurait adopté un dispositif similaire en 2004, constitue lui aussi une référence de la proposition de loi.

La réalité britannique est loin d'être aussi linéaire, et présente aujourd'hui encore de nombreuses contradictions. Tout d'abord, le dispositif invoqué ne concerne aujourd'hui que les étudiants anglais et gallois. La décentralisation des pouvoirs, accordée à l'Irlande du Nord, au Pays de Galles et à l'Écosse, en 1999, dans le cadre de la «devolution $»^{6}$, a introduit de profondes divergences ${ }^{7}$ sur l'ensemble des questions éducatives au sein du Royaume-Uni. D'autre part, et c'est l'objet de cet article, le remplacement de la gratuité et des bourses ${ }^{8}$ par les frais d'inscription et les prêts étudiants, et les objectifs politiques qui lui sont associés, ont commencé à se concrétiser à l'occasion de l'expansion de l'enseignement supérieur, organisée par les conservateurs, dès 1987. Cette recomposition de la distribution des ressources monétaires dévolues aux étudiants s'inscrit dans une politique publique d'enseignement supérieur initiée par les conservateurs et poursuivie par les gouvernements de Tony Blair, à partir de 1997. Au-delà des éléments de rupture qui la traversent, cette politique publique a eu pour objectif constant, depuis 1987, l'augmentation des effectifs étudiants et l'élargissement de l'accès à l'enseignement supérieur aux étudiants dits « non traditionnels », issus des groupes socio-économiques défavorisés (classes populaires, femmes célibataires, minorités ethniques...). Si cette volonté d'expansion du système éducatif n'est pas nouvelle, les impératifs économiques (attractivité du territoire pour les capitaux, employabilité de la main-d'œuvre, recherche et développement...) qui lui sont associés le sont davantage (Deer, 2004). Ils ont contribué à mettre en doute

\footnotetext{
${ }^{6}$ La « devolution » dote les parlements gallois, écossais et nordirlandais de nouveaux domaines de compétences législatives, notamment en matière d'éducation.

7 À titre d'exemple, l'Écosse maintient toujours la gratuité pour les étudiants domiciliés sur son territoire.

${ }^{8}$ Le système des « bourses obligatoires ", géré par les autorités locales et supprimé en 1999, ne fera l'objet que de quelques allusions au sein de cet article. Il trouve son origine dans les politiques éducatives adoptées après-guerre et a connu un développement remarquable sous plusieurs points (montant des bourses, nombre de bénéficiaires, dépenses publiques mobilisées...), des années 60 à sa suppression.
}

la nature et la réalité de la politique d'accès préconisée depuis 1987.

En considérant les frais d'inscription et les prêts étudiants comme des éléments fondamentaux de cette expansion, et en confrontant leurs effets sur la nature et l'origine des revenus étudiants à leurs objectifs politiques, nous entendons mettre en question la capacité des frais d'inscription et des prêts étudiants à reconfigurer, dans le temps, les réalités sociales étudiantes. En effet, dans quelle mesure parviennent-ils à modifier les rapports que les étudiants anglais entretiennent avec les études supérieures, leur famille ou le marché du travail et quel est le poids du groupe socio-économique d'appartenance dans ces évolutions?

Le rapport Dearing, publié en 1997, les livres blancs sur l'enseignement supérieur, publiés en 1987, 1991 et 2003, illustrent les ruptures et les continuités des objectifs assignés à l'enseignement supérieur par les conservateurs et les travaillistes, depuis une vingtaine d'années ( $1^{\text {re }}$ Partie). L'élargissement et l'augmentation des effectifs étudiants ont été financés via la distribution de plus en plus massive de prêts et autres ressources afin de soulager les étudiants des augmentations successives des frais d'inscription ( $2^{\mathrm{e}}$ Partie). Bien que l'enseignement supérieur britannique paraisse aujourd'hui plus accessible, un mouvement général et durable conditionne désormais l'accès aux études supérieures à un endettement croissant et à l'exercice d'une activité salariée ( $3^{\mathrm{e}}$ Partie). La composition des revenus étudiants en fonction des groupes socio-économiques d'appartenance révèle par ailleurs la formation de nouvelles inégalités entre étudiants (4 $4^{\mathrm{e}}$ Partie).

\section{HAUSSE DES EFFECTIFS, ACCÈS ÉLARGI ET ARTICULATION AUX IMPÉRATIFS NATIONAUX}

Les livres blancs « Enseignement supérieur : relever le défi » (DES, 1987), «Enseignement supérieur : une nouvelle structure » (DES, 1991) et «Le futur de l'enseignement supérieur » (DES, 2003) ont été l'occasion, pour les décideurs politiques britanniques, d'exprimer les objectifs qu'ils assignaient à l'enseignement 
supérieur. «L'enseignement supérieur dans une société de la connaissance » est un rapport publié en 1997 par le comité Dearing, comité composé d'experts reconnus par l'ensemble de la classe politique parlementaire. Il était chargé de proposer de nouvelles orientations pour l'enseignement supérieur qui tiennent compte des évolutions économiques et sociales, de l'émergence d'une société de la connaissance et de la nécessité, pour le Royaume-Uni, d'être en bonne place dans la compétition mondiale. Ces documents mettent en lumière les accords et les désaccords entre travaillistes et conservateurs quant aux motivations du processus d'expansion de l'enseignement supérieur.

\section{Des effectifs qui augmentent, un enseignement supérieur qui se développe}

La nouvelle politique publique, adoptée en 1987, avait pour premier horizon la fin des années 2000 et l'accès d'un tiers des effectifs de la classe des 18-30 ans à l'enseignement supérieur. L'objectif fut atteint et laissa la place, dans un second temps, à l'objectif travailliste de l'accès d'une moitié de la classe d'âge en 2010. Si la capacité du Royaume-Uni à atteindre ce nouvel objectif est sérieusement mise en doute par la récente stagnation des effectifs, la hausse du nombre d'étudiants n'en reste pas moins remarquable. Les effectifs ont doublé en près de vingt ans et se situent dorénavant à la hauteur des autres pays de l'OCDE (Organisation de coopération et de développement économiques).

Certes, l'augmentation des effectifs a davantage concerné les étudiants étrangers. Néanmoins, elle ne s'est pas réalisée au détriment des études à temps plein qui ont attiré tout autant que les études à temps partiel, en conservant une place largement majoritaire au sein des effectifs globaux ${ }^{9}$. Cette expansion est d'autant plus remarquable qu'elle s'est opérée à dépenses publiques constantes au cours des années 90 , tendance que les travaillistes vont s'attacher à remettre en cause au début de leur mandat, dans la lignée du rapport Dearing. Plus généralement, depuis une vingtaine d'années, c'est incontestablement la croissance des revenus privés des universités qui a permis de financer l'expansion (Carpentier, 2004), les frais d'inscription y occupant une part croissante et déterminante.

L'expansion nécessitait par ailleurs d'élargir les capacités d'accueil des établissements. La Loi sur l'enseignement supérieur de 1992 a ainsi autorisé les établissements polytechniques, traditionnellement dévolus à un enseignement plus professionnalisé que les universités, à se transformer en universités, simplifiant considérablement la structure de l'enseignement supérieur britannique. Les organismes de recrutement, les conseils publics de financement et les agences chargées d'évaluer la qualité des établissements ont été unifiés à cette occasion.
9 Les études à temps partiel permettent à l'individu d'allonger et de modifier la temporalité d'obtention du diplôme de l'enseignement supérieur en fonction de ses autres contraintes de travail. Cette modalité de validation des études supérieures est née dans les années 60 et elle connaît un certain succès depuis la fin des années 80. Elle concerne depuis environ $40 \%$ des effectifs, essentiellement des salariés.

\section{Tableau 1}

Évolution du nombre d'étudiants inscrits et domiciliés au Royaume-Uni, de 1988 à 2007, selon leurs modalités d'études

\begin{tabular}{|c|c|c|c|c|c|c|}
\hline & $1988 / 89$ & $1992 / 93$ & $1995 / 96$ & $1998 / 99$ & $2004 / 05$ & $2007 / 08$ \\
\hline Étudiants (en milliers) & 918 & 1304 & 1720 & 1846 & 2288 & 2306 \\
Proportion à temps plein & $68,2 \%$ & $71,6 \%$ & $64,4 \%$ & $63,9 \%$ & $60,8 \%$ & $64,2 \%$ \\
Proportion à temps partiel & $31,8 \%$ & $28,4 \%$ & $35,6 \%$ & $36,1 \%$ & $39,2 \%$ & $35,8 \%$ \\
\hline Étudiants étrangers (en milliers) (*) & 24,3 & 93,7 & 196,3 & 219,3 & 297,1 & 341,8 \\
Proportion de l'ensemble & $2,6 \%$ & $7,1 \%$ & $17,4 \%$ & $17,9 \%$ & $13 \%$ & $14,8 \%$ \\
\hline
\end{tabular}

Source : Higher education statistics agency.

(*) : Uniquement au niveau undergraduate en 1988. Sauf s'ils sont ressortissants de l'Union européenne, les étrangers paient des frais d'inscription beaucoup plus élevés et ne bénéficient pas des ressources accordées aux étudiants britanniques. 


\section{Articuler l'impératif de l'accès aux études supérieures aux autres impératifs nationaux}

Si les trois livres blancs affichent comme objectif principal l'élargissement de l'accès aux études supérieures, ils l'articulent, dès leur introduction, à d'autres impératifs nationaux. Le rapport Dearing (1997), issu d'une réflexion générale, consensuelle et indépendante des impératifs gouvernementaux de l'époque, illustre l'accord des travaillistes et des conservateurs concernant les principaux objectifs assignés aux politiques publiques d'enseignement supérieur depuis 1987 :

« Le Comité a du considérer [...] les principes suivants : il devrait y avoir une participation maximale dans l'enseignement supérieur initial [...] et dans la formation tout au long de la vie [...] pour prendre en compte les besoins futurs des individus, de la nation et du marché du travail ; les étudiants devraient pouvoir choisir au sein d'une large variété de cours, d'institutions, de modalités et de lieux d'études ; [...] L'efficacité de l'enseignement et de l'apprentissage devrait être augmentée ; l'apprentissage devrait être de plus en plus sensible aux besoins des employeurs et inclure le développement de compétences générales largement valorisées dans l'emploi ; [...] Les modalités du système de soutien étudiant devraient être justes et transparentes et soutenir les principes précédents ; [...] le rapport qualitélprix devrait s'imposer dans l'utilisation des ressources. » (Dearing, 1997, p. 3).

La suite de l'avant-propos est quant à elle consacrée au contexte futur. Elle permet au comité d'évoquer l'exacerbation de la compétition internationale en matière de croissance économique, de personnels et d'étudiants (p. 4).

L'articulation d'impératifs liés à l'expansion de l'enseignement supérieur, à la justice sociale, aux besoins en main-d'œuvre qualifiée, à la qualité des universités britanniques dans la compétition internationale et à la capacité du territoire à attirer et valoriser les capitaux, constitue ainsi selon nous le " fond commun » du discours des décideurs politiques depuis une vingtaine d'années. Les transformations de la population étudiante générées par ces politiques nous permettront par la suite d'en dire davantage sur ce qui fait leur continuité.

\section{LE DÉVELOPPEMENT DE NOUVELLES RESSOURCES}

L'introduction de prêts étudiants complémentaires, en 1990, a été légitimée dans un livre blanc, publié en 1988, et intitulé «Des prêts complémentaires pour les étudiants ». Ce document détaille aussi les modalités de distribution de la ressource. Face au refus des entreprises commerciales (banques...) d'assurer la distribution de prêts à taux préférentiel, le gouvernement britannique sera contraint de créer un nouvel organisme à même d'assumer cette nouvelle fonction de l'État, la « Student loans company » (SLC). Cette agence alloue des subventions publiques qui ont pris une nouvelle envergure depuis l'arrivée des travaillistes.

\section{L'essor des agences gouvernementales au détriment des autorités locales}

La création, en 1990, de la SLC par le gouvernement conservateur britannique a marqué la rupture avec la politique antérieure des mandatory awards, un système de bourses géré par les autorités locales et garantissant à la majorité des étudiants britanniques la gratuité des études supérieures et le versement de bourses dédiées aux autres dépenses de vie courante (Casta, 2007). Si la gestion des autorités locales revient à des représentants politiques locaux élus au suffrage universel, la SLC est une agence financée par le gouvernement mais au fonctionnement indépendant du ministère ou de toute autre institution politique. Le rôle de la SLC a en effet été accru, depuis 1997, puisqu'elle s'est vue confier la distribution de nouvelles ressources et qu'une nouvelle agence, l' « Office for fair access » (OFFA), a été lancée en 2006 afin de mettre en place, dans les établissements universitaires, des politiques locales de soutien aux étudiants.

\section{Une nouvelle ressource pour l'entretien des étudiants...}

Dans le livre blanc publié en 1988, l'introduction des prêts étudiants est vue comme un moyen de concilier expansion de l'enseignement supérieur et volonté de stabiliser les dépenses publiques. Ces dernières sont 
à l'époque considérablement alourdies par le système des mandatory awards. Associé à l'expansion à venir, ce système de bourses risquait de favoriser un envol des dépenses publiques. Les conservateurs entérinent donc le début de la distribution de prêts dont les montants compenseront les baisses successives et annoncées du montant des bourses. L'introduction des prêts est aussi l'occasion d'entériner un partage plus équitable du coût des études supérieures, ainsi que de socialiser les étudiants à de nouveaux comportements ayant trait à leur « conscience économique et à leur autonomie » (DES, 1988 ; p. 1). L'argumentaire des conservateurs est très pragmatique. Les étudiants doivent être soutenus pour leur entretien, les parents et les contribuables participent déjà largement à cet entretien et il se trouve que le salaire des diplômés est en moyenne un quart plus élevé sur le marché du travail. Ces trois faits légitiment la création de prêts dont le remboursement sera effectué une fois les études terminées.

\section{... mais de nouvelles dépenses d'inscription}

Si le partage des coûts d'entretien à la charge des étudiants fut soutenu ouvertement par les conservateurs, celui du coût des études fut imposé avec beaucoup moins de transparence. Le gouvernement Major a laissé, de 1990 à 1997, les établissements d'enseignement supérieur augmenter et différencier les frais d'inscription des étudiants à temps plein, dans un contexte de stabilisation des dépenses publiques affectées aux universités et de gel du montant de la ressource accordée aux étudiants boursiers pour payer leurs frais d'inscription.

Ces hausses par établissement et par cursus recevront un discret assentiment du gouvernement à l'occasion du livre blanc de 1991 (DES, 1991 ; chapitre 2). Les travaillistes vont confirmer cet accroissement des charges liées aux études. Dès 1998, ils instaurent en effet des frais d'inscription et redonnent au gouvernement le pouvoir de les déterminer. Les frais sont alors de $1000 £(1569 €)$. Ils sont soumis à une modulation par tiers ${ }^{10}$ pour l'ensemble des étudiants à temps plein. En totale contradiction avec les mesures travaillistes, les mandatory awards sont supprimés.

Pour les remplacer, le gouvernement crée, en 1999, le « nouveau système de soutien aux étudiants". Ce système s'appuie exclusivement sur les prêts de la SLC, dont les montants sont considérablement augmentés en 1999, et dont le quart est soumis à conditions de ressources. Ces montants et les frais d'inscription seront réévalués chaque année à hauteur de l'inflation, de 1998 à 2005 (passant de $1000 £$ à $1250 £$ pour les frais d'inscription). La loi sur l'enseignement supérieur de 2004 introduit une nouvelle rupture. Les universités auront, à partir de 2006, la possibilité d'établir leurs frais d'inscription jusqu'à $3000 £(3750 €)$. La quasi-totalité des universités (DES, 2006) choisit alors le plafond maximal, s'engageant en retour auprès de l'OFFA à mettre en place des bourses à destination des étudiants défavorisés. Deux types de prêts seront désormais accessibles aux étudiants à temps plein et leur montant représente une augmentation sans précédent. Tous les étudiants pourront en effet souscrire un prêt pour frais d'inscription s'élevant à $3000 £$ et un prêt pour entretien pouvant s'élever à $6000 £(7500 €)$ (tableau 3). Une nouvelle bourse sous conditions de ressources, intitulée « bourse d'entretien », est aussi mise en place.

\footnotetext{
${ }^{10}$ Seul le tiers le plus favorisé des étudiants payait la totalité de cette somme. Le tiers le moins favorisé en était exempté et le tiers intermédiaire n'en payait qu'une partie, modulée en fonction des ressources parentales déclarées.
}

Tableau 2

Évolution des dépenses annuelles moyennes consacrées aux études(*) de 1992 à 2004 pour les étudiants à temps plein $\left(£\left({ }^{* *}\right) 2004\right)$

\begin{tabular}{|c|c|c|c|c|}
\hline & $1992 / 93$ & $1995 / 96$ & $1998 / 99$ & $2004 / 05$ \\
\hline Dépenses études & $325(429 €)$ & $602(795 €)$ & $893(1178 €)$ & $1980(2614 €)$ \\
\hline
\end{tabular}

Source : SIES (1993, 1996, 2000, 2006).

(*) : Les dépenses liées aux études comprennent d'autres postes que les frais d'inscription (matériel, transport...). Les frais d'inscription constituent toutefois les deux tiers de la dépense en 2004 et sont le principal vecteur de la hausse observée.

$(* *)$ : Les montants donnés par la suite en euros correspondent à la valeur de l'euro au $1^{\text {er }}$ janvier 2010. 
Ses plafonds d'attribution la destinent à une minorité de la population et son montant maximal est, en 2006, de $2700 £(3375 €)$.

Une autre rupture travailliste est notable. Dès 1998, le gouvernement décide, notamment sous l'influence du rapport Dearing, de créer de nouvelles ressources à disposition des étudiants à temps partiel. C'est une évolution sans précédent puisque, malgré leur nombre important, ils ont historiquement toujours été moins bien lotis que les étudiants à temps plein. Le Livre blanc de 2003 leur accorde également la possibilité de bénéficier de bourses d'entretien et de bourses d'inscription. Ces dernières succèdent aux prêts de $500 £$ (763€) qui pouvaient leur être accordés depuis 1999. Ces bourses restent d'un montant fort modeste ( $250 £$ maximum, soit $330 €$ ), comparées aux autres bourses, et elles ne bénéficient qu'à une petite minorité, la tranche la plus défavorisée des étudiants à temps partiel (UUK, 2006).

Le document « Vers une seule bourse combinée pour l'enseignement supérieur », annexé au livre blanc de
2004, contribue à justifier l'adhésion des travaillistes à la poursuite du développement concomitant des frais d'inscription et de l'endettement étudiant, ainsi que la création des nouvelles ressources. Ce développement vise à garantir une augmentation durable et équitable des ressources à disposition des établissements d'enseignement supérieur. La volonté de justice sociale conduit aussi les travaillistes à reprendre l'argument du surplus de salaire des diplômés par rapport aux non-diplômés.

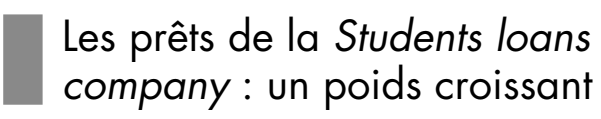

Le livre blanc de 1988 et la Loi sur les prêts étudiants, votée en 1990, fixent les orientations générales de la SLC. C'est une institution non lucrative au budget voté par le Parlement et tenue de rendre des comptes au Secrétaire d'État en charge de l'Enseignement supérieur. Le Secrétaire fixe, quant à lui, le montant maximal du prêt, les conditions de son remboursement et prend des dispositions pour faire face aux défauts de paiement temporaires ou définitifs. L'institution

Tableau 3

Évolution du montant des prêts pour les étudiants à temps plein anglais depuis 1990 (£ 2006)

\begin{tabular}{|c|c|c|c|}
\hline Année & Lieux de résidence (*) & Prêts entretien & Prêts inscription \\
\hline 1990/91 & $\begin{array}{l}\text { Décohabitant à Londres } \\
\text { Domicile d'origine } \\
\text { Décohabitant hors de Londres }\end{array}$ & $\begin{array}{l}719(899 €) \\
516(645 €) \\
657(821 €)\end{array}$ & n.e. \\
\hline $1996 / 97$ & $\begin{array}{l}\text { Décohabitant à Londres } \\
\text { Domicile d'origine } \\
\text { Décohabitant hors de Londres }\end{array}$ & $\begin{array}{l}2588(3235 €) \\
1602(2002 €) \\
2092(2615 €)\end{array}$ & n.e. \\
\hline 1998/99 & $\begin{array}{l}\text { Décohabitant à Londres } \\
\text { Domicile d'origine } \\
\text { Décohabitant hors de Londres }\end{array}$ & $\begin{array}{l}3796(4745 €) \\
2806(3507 €) \\
3301(4126 €)\end{array}$ & n.e. \\
\hline $2004 / 05$ & $\begin{array}{l}\text { Décohabitant à Londres } \\
\text { Domicile d'origine } \\
\text { Décohabitant hors de Londres }\end{array}$ & $\begin{array}{l}5472(6840 €) \\
3426(4282 €) \\
4330(5412 €)\end{array}$ & n.e. \\
\hline \multirow[t]{2}{*}{ 2006/07 } & $\begin{array}{l}\text { Décohabitant à Londres } \\
\text { Domicile d'origine } \\
\text { Décohabitant hors de Londres }\end{array}$ & $\begin{array}{l}6170(7712 €) \\
3415(4269 €) \\
4405(5506 €)\end{array}$ & $\begin{array}{l}3000(3750 €) \\
3000(3750 €) \\
3000(3750 €)\end{array}$ \\
\hline & Évolution 1990/2006 & $\begin{array}{l}+758 \% \\
+562 \% \\
+570 \%\end{array}$ & \\
\hline
\end{tabular}


est tenue de rembourser les sommes versées par les pouvoirs publics.

Les prêts distribués sous la période conservatrice étaient de type hypothécaire. Ainsi, une fois les études terminées, le remboursement annuel dépendait en premier lieu du montant emprunté au cours des études. Plusieurs éléments rendaient ce remboursement particulièrement pesant pour les emprunteurs. Dès la fin de l'année universitaire, la dette était susceptible d'être remboursée à la SLC. Le remboursement pouvait être échelonné et repoussé mais les conditions de ressources pour bénéficier de ce dispositif étaient fixées par l'agence à un niveau particulièrement bas. Enfin, formellement, aucun intérêt n'était dû mais dès que l'échéance était repoussée d'une année, la dette était réévaluée en fonction de l'indice des prix à la consommation, qui pouvait s'avérer à l'époque supérieur aux taux d'intérêt proposés par les banques commerciales (CVCP, 1989). Ces prêts étaient accordés à tous les étudiants à temps plein inscrits dans les cursus du premier niveau de l'enseignement supérieur (undergraduates) jusqu'à l'âge de 50 ans. Trois niveaux de montant dépendant du lieu de résidence avaient été créés. La dette était effacée 25 ans après la souscription du premier emprunt, après 50 ans (60 ans pour ceux ayant commencé leur cursus après 40 ans) et en cas de décès.

Au-delà de la réévaluation des montants disponibles, les travaillistes ont apporté quelques changements au dispositif. Le changement principal réside dans les modalités de remboursement. Le système hypothécaire est remplacé par un système fiscal. Le remboursement est dorénavant adressé aux autorités fiscales britanniques une fois les études supérieures terminées. Le dispositif apparait alors plus transparent puisque les lois garantissent désormais que le remboursement démarre à partir d'un seuil de revenu annuel du diplômé (15000 $£^{11}$ soit $17343 €$ ) et d'un prélèvement fiscal (équivalent à $9 \%$ de la tranche dépassant les $15000 £$ ) clairement déterminés.

Lancés en 1990, les prêts d'entretien (maintenance loans) de la SLC ont fini par être distribués à la quasitotalité de la population éligible ( $80 \%$ en 2004). Les montants que les étudiants à temps plein sont

11 L'une des grandes mesures de la Loi sur l'enseignement supérieur de 2004 a été de faire passer ce seuil de $10000 £$ à $15000 £$. autorisés à solliciter sont quant à eux six à huit fois plus élevés en 2006 qu'en 1990 ( $c f$. tableau 3). La hausse est encore plus significative si les nouveaux prêts d'inscription de $3000 £$ ( $3750 €)$ sont pris en compte. Les dépenses publiques consacrées aux prêts ont continué de croître lors des mandats travaillistes. Les nouvelles dispositions ont multiplié par cinq les dépenses publiques mobilisées à cette occasion, de 1997 à 2006, en Angleterre et au Pays de Galles (Casta, 2007). Notons que les remboursements des prêts des diplômés ne couvrent actuellement qu'environ $20 \%$ de la dépense publique occasionnée chaque année par la distribution de ces prêts publics ${ }^{12}$.

\section{UN ACCÈS GÉNERALISÉ MARQUÉ PAR L'ENDETTEMENT ET L'EMPLOI}

L'expansion de l'enseignement supérieur génère un double mouvement. Si elle maintient, tout en les recomposant, les divisions entre groupes socio-économiques, elle accompagne un mouvement général et durable qui rend la condition financière des étudiants plus homogène. Par les politiques publiques d'enseignement supérieur menées depuis une vingtaine d'années, les décideurs politiques britanniques sont parvenus à imposer un mouvement touchant aujourd'hui la quasitotalité des étudiants. Les étudiants anglais présentent aujourd'hui de nouvelles caractéristiques sociologiques. Leur statut et leur accès à l'enseignement supérieur ont désormais pour condition un endettement croissant et la pratique plus ou moins continue d'une activité salariée ${ }^{13}$. Ce mouvement est d'autant plus remarquable qu'il concerne une population de plus en plus importante et qu'il n'a pas été remis en cause par le changement de gouvernement en 1997.

\footnotetext{
${ }^{12}$ La proportion ne cesse toutefois d'augmenter depuis la création de la SLC. À titre d'exemple, elle n'était que de $10 \%$ lors de l'année universitaire 1996/1997 (SLC, 1998 et 2007).

13 Plusieurs éléments statistiques disponibles dans les enquêtes statistiques laissent à penser que l'emploi étudiant concurrence effectivement le temps des études, et qu'il est vécu par une bonne partie des étudiants comme un facteur particulièrement contraignant. À titre d'exemple, en 2004, ils sont respectivement $39 \%$ et $52 \%$ d'étudiants à temps plein et à temps partiel à estimer que leur emploi affecte leurs études (santé, bien-être, résultats, travail personnel...) (SIES, 2006).
} 


\section{Des étudiants à temps plein employés et endettés}

Un mouvement général d'endettement et de recours au salariat s'amorce chez les étudiants à temps plein, dès l'introduction des prêts en 1990. Le graphique 1 montre que la baisse progressive du soutien parental, et surtout des bourses, est compensée par l'augmentation des revenus salariaux et des prêts de la SLC, à hauteur de $14 \%$ chacun, la part du crédit commercial augmentant également.

Le mouvement est similaire sous l'ère travailliste. À la baisse des bourses et de la contribution parentale répond une hausse du poids moyen des prêts publics et de l'activité salariée. Ces deux derniers éléments voient leur poids passer de $42 \%$ à $55 \%$. Par ailleurs, $56 \%$ des étudiants à temps plein travaillent en 2004 durant l'année universitaire. Si la proportion était plus importante (62\%) en $1998^{14}$, d'autres changements alimentent la thèse d'une place croissante accordée au salariat. Ils sont $39 \%$ à exercer un emploi continu durant l'année universitaire en 2004, contre $23 \%$ en 1998. Le temps de travail moyen augmente et le salaire moyen perçu durant l'année universitaire a plus que doublé en valeur réelle. Enfin, ils sont $86 \%$

\footnotetext{
${ }^{14}$ La hausse du nombre d'étudiants constatée dans le même temps (tableau 1) et les chiffres syndicaux (TUC, 2008) nuancent fortement cette baisse relative.
}
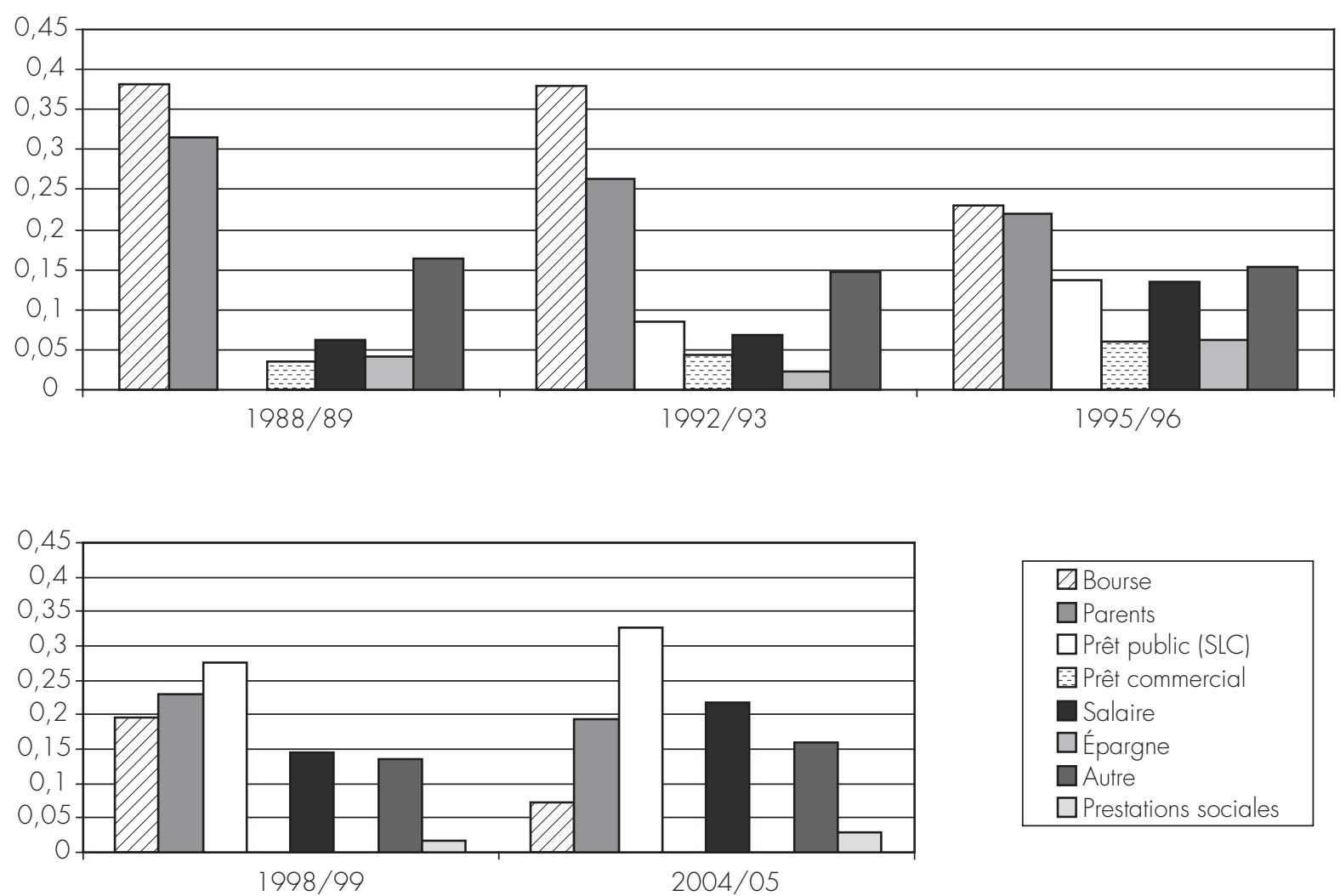

\begin{tabular}{|l|}
\hline$\square$ Bourse \\
$\square$ Parents \\
$\square$ Prêt public (SLC) \\
Prêt commercial \\
$\square$ Salaire \\
$\square$ Épargne \\
$\square$ Autre \\
$\square$ Prestations sociales \\
\hline
\end{tabular}

Source : SIES 2000 et 2006

(*) : Pour rappel, la convention statistique du revenu change en 1998 et exclut l'épargne et le prêt commercial de la mesure du revenu. Ceux-ci font l'objet d'un traitement à part dans les enquêtes. Les prestations sociales sont, depuis 1998, l'objet d'un poste spécifique. Ce n'était pas le cas avant, elles étaient intégrées au poste « autre », tout comme les aides financières des établissements, ou celles venant des amis ou des autres membres de la famille. Le poste « épargne » renvoie aux retraits d'épargne effectués par les étudiants. Le poste « bourse » inclut, avant 1998, l'ensemble des sommes versées au titre des mandatory awards, ces dernières étant supprimées en 1998, les deux dernières enquêtes incluent dans le poste « bourse » le paiement sous conditions de ressources des frais d'inscription. 
(contre $82 \%$ en 1998 et $71 \%$ en 1995) à exercer un emploi durant les vacances d'été ${ }^{15}$. Ils sont $22 \%$ (contre $27 \%$ en 1998) à recourir à des prêts bancaires dont le montant moyen a doublé depuis 1998. La dette moyenne déclarée a elle aussi doublé.

Il semble par ailleurs que les étudiants à temps plein se salarient dans des conditions de rémunération au-dessous de la moyenne britannique. Le salaire horaire moyen déclaré lors des enquêtes confirme tout d'abord cette propension des étudiants à occuper des emplois moins bien rémunérés. Une brochure publiée en 2008, dans le cadre d'un partenariat entre le syndicat étudiant (NUS) et l'organisation syndicale britannique (TUC), confirme ce diagnostic. Plus des deux tiers des étudiants à temps plein se concentrent dans deux secteurs d'activité, l'hôtellerie-restauration pour plus du quart, et le commerce pour plus de $40 \%$, deux secteurs d'activité présentant un salaire moyen de $25 \%$ à $50 \%$ plus bas que celui en vigueur dans les autres secteurs.

\section{Un accès toujours fondé sur l'emploi pour les études à temps partiel}

Les études à temps partiel ont elles aussi bénéficié de l'expansion de l'enseignement supérieur depuis 1987 (tableau 1). Leur reconnaissance publique et la remise en cause des inégalités financières qui les touchaient durant les mandats conservateurs sont annoncées dans le rapport Dearing (1997). Les gouvernements travaillistes s'efforceront par la suite de les intégrer au système de soutien étudiant, par le biais de prêts, puis de bourses.

Les enquêtes de 1999 et de 2005 détaillent la composition des revenus de ces étudiants à temps partiel. La majeure partie de ces revenus (à hauteur de $80 \%$ ) continue de provenir de leur activité salariée. Ils sont plus de $80 \%$ à exercer ce type d'activité durant l'année universitaire. La hausse des dépenses liées aux études (à hauteur de $20 \%$ ) semble, malgré les aides travaillistes, les avoir contraints à recourir davantage au crédit commercial. Ils sont plus nombreux à sous-

${ }^{15}$ L'impact financier de cette activité estivale est loin d'être négligeable. Elle est traitée à part dans chacune des enquêtes pour des raisons méthodologiques; les auteurs de l'étude de 2004 estiment que cette activité double les revenus salariaux moyens perçus chaque année par les étudiants à temps plein. crire un prêt commercial, le taux passant de $50 \%$ à $56 \%$, et le montant moyen des prêts bancaires souscrits ayant augmenté de $50 \%$. La dette moyenne déclarée augmente dans les mêmes proportions.

\section{UN ACCÈS MASSIFIÉ MAIS DIFFÉRENCIÉ ?}

L'expansion de l'enseignement supérieur a engendré une recomposition des inégalités entre groupes socio-économiques dans l'enseignement supérieur. Sa capacité à bénéficier à toutes les classes sociales a été vivement mise en doute. Les « Enquêtes sur les revenus et les dépenses des étudiants » (SIES) permettent d'évaluer le poids et l'évolution des inégalités entre groupes socio-économiques dans la recomposition des revenus étudiants initiée en 1990.

\section{Un accès élargi depuis $1997 \ldots$}

Les responsables travaillistes ont pour habitude de rétorquer à leurs détracteurs que leur nouvelle politique de soutien aux étudiants n'a pas augmenté les inégalités sociales en matière d'accès et qu'un jeune ayant terminé avec succès ses études secondaires a toutes les chances d'entrer à l'université, quel que soit son milieu socio-économique d'origine (DES, 2003). Les évolutions récentes de deux indicateurs statistiques sont régulièrement mobilisées par les travaillistes. D'une part, la composition sociale des étudiants à temps plein entrant dans l'enseignement supérieur lors d'une année universitaire est restée globalement stable sous l'ère travailliste. Ce n'était pas le cas sous les gouvernements conservateurs où les étudiants issus des milieux défavorisés occupaient une place de moins en moins conséquente. D'autre part, le taux de participation à l'enseignement supérieur d'une classe d'âge augmente à un rythme équivalent dans tous les groupes socio-économiques. Le rythme des groupes inférieurs était là encore moins soutenu sous l'ère conservatrice. Dans un contexte de hausse des effectifs, et comparée aux résultats de leurs prédécesseurs (Dearing, 1997), l'évolution de ces deux indicateurs valorise les politiques travaillistes. 
L'argumentaire travailliste a suscité une contre-expertise sociologique et économique particulièrement riche (Callender, 2002 ; Ball, David, Davies et Reay, 2001 ; Pennel et West, 2005 ; Stanton, 2008) ${ }^{16}$. Ces travaux se sont particulièrement intéressés à l'impact de l'introduction des frais d'inscription et des prêts étudiants sur les groupes socio-économiques les plus défavorisés et les minorités ethniques. Ils mettent notamment en avant le poids de ces nouvelles contraintes financières dans le choix des établissements, leur capacité à renforcer les hiérarchies existantes dans l'enseignement supérieur britannique, la propension plus importante de ces populations à s'endetter et à travailler durant les études, ainsi que leur incapacité à souscrire sans crainte un emprunt d'autant plus incertain qu'il accompagne des contraintes économiques plus immédiates. Un état des lieux de ces inégalités et de leur évolution dans la composition des revenus étudiants est proposé par la suite.

\section{... au prix de concessions} plus dures des classes populaires

Le graphique 2 illustre la recomposition, sous le mandat conservateur, des revenus des étudiants en fonction de leur groupe socio-économique d'appartenance. Le poids de l'activité salariée s'est accru. Cette hausse est particulièrement marquée de 1992 à 1995 ; elle porte la proportion entre 10 et $15 \%$ et touche toutes les classes. La hausse est toutefois plus marquée dans les classes inférieures (IV et V). Le constat est le même pour la part des prêts de la SLC. Les crédits commerciaux se développent quant à eux lentement, jusqu'à occuper un peu plus de $5 \%$ du revenu étudiant. Ce dernier mouvement traverse toutes les classes, à l'exception notable de la classe supérieure.

En ce qui concerne les évolutions observables sous les mandats travaillistes, elles sont moins fines en raison des regroupements de classes opérés par les auteurs des deux enquêtes de 1999 et 2005. Les comparaisons entre groupes socio-économiques portent uniquement sur trois catégories. Le graphique 3 révèle que l'impact de l'origine socio-économique reste marginal

\footnotetext{
${ }^{16}$ La richesse du débat s'est encore accrue ces dernières années avec les nouvelles hausses des frais d'inscription mises en place en 2006 et celles annoncées pour 2010 (NUS, 2007).
}

en matière de prêts publics et a même tendance à se réduire si l'on considère l'activité salariée, même si la catégorie inférieure continue d'être plus touchée par le phénomène. En somme, de prime abord, ce tableau des conséquences des politiques menées par les travaillistes semble confirmer la capacité de leur dispositif à limiter, voire réduire, les inégalités entre groupes socio-économiques quant à la part de l'endettement et du salaire dans les revenus étudiants.

Toutefois, une analyse plus fine de la composition du revenu et de ses sources tend à confirmer la persistance et l'accroissement des inégalités. Tout d'abord, la hausse des dépenses liées aux études a davantage touché la catégorie inférieure. Le résultat est qu'en 2004, les dépenses s'accroissent au fur et à mesure que l'on descend dans la hiérarchie sociale. Ce n'était pas le cas en 1998. Le salariat, durant l'année universitaire, reste plus fréquent dans les groupes inférieurs. Le temps de travail continue de s'allonger au fur et à mesure que l'on descend dans la hiérarchie sociale. Si les étudiants des classes populaires travaillent plus longtemps, le revenu moyen issu du salariat a tendance à s'égaliser, le groupe supérieur touchant à peine moins que le groupe inférieur en 2004. Cela suggère fortement des inégalités de salaire, les étudiants issus du groupe supérieur parvenant à se salarier dans des emplois mieux rémunérés.

Si l'origine socio-économique semble toujours avoir peu d'impact sur la propension des étudiants à souscrire un prêt public et sur le montant de ce prêt, ce n'est pas le cas des crédits commerciaux. Le montant emprunté en 2004 augmente au fur et à mesure que l'on descend dans la hiérarchie des groupes ${ }^{17}$. Il est en moyenne de $1469 £$ (1939€) dans la classe inférieure, soit $60 \%$ de plus par rapport à la classe supérieure.

Ces inégalités constatées quant au montant moyen du crédit souscrit par l'étudiant, au moment de l'enquête, n'existaient pas lors de l'enquête de 1993. Même si les différences méthodologiques des deux enquêtes peuvent nuancer la comparaison, les deux enquêtes indiquent que le montant déclaré par l'étudiant a été multiplié par quatre en valeur réelle pour les classes

\footnotetext{
${ }^{17}$ Ce besoin de recourir aux prêts commerciaux peut paraître étonnant au regard des sommes déjà mises à disposition chaque année par la SLC. Claire Callender (2002) considère que ces sommes ne sont effectivement pas suffisantes pour combler les besoins de certains étudiants ayant des charges familiales.
} 
Graphique 2

Poids des parents, du prêt commercial, du salaire et du prêt public (Student loans company) dans le revenu moyen des étudiants à temps plein selon les classes sociales en 1988, 1992 et 1995

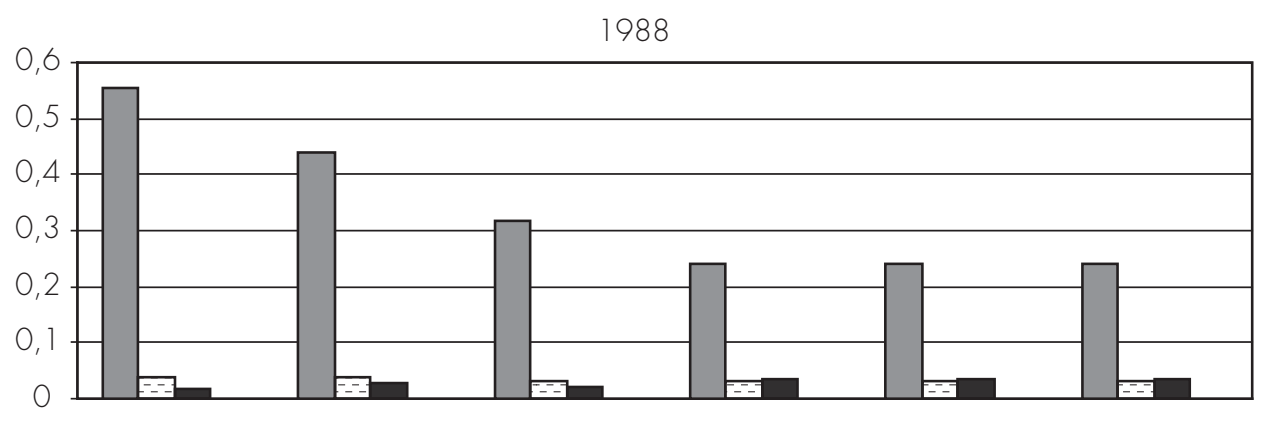

1992

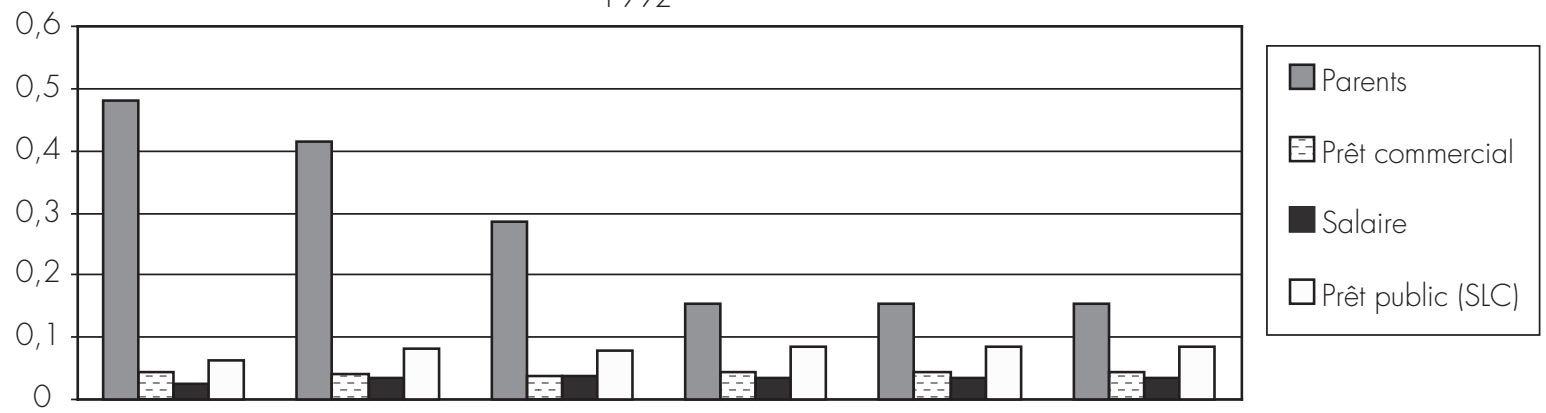

1995

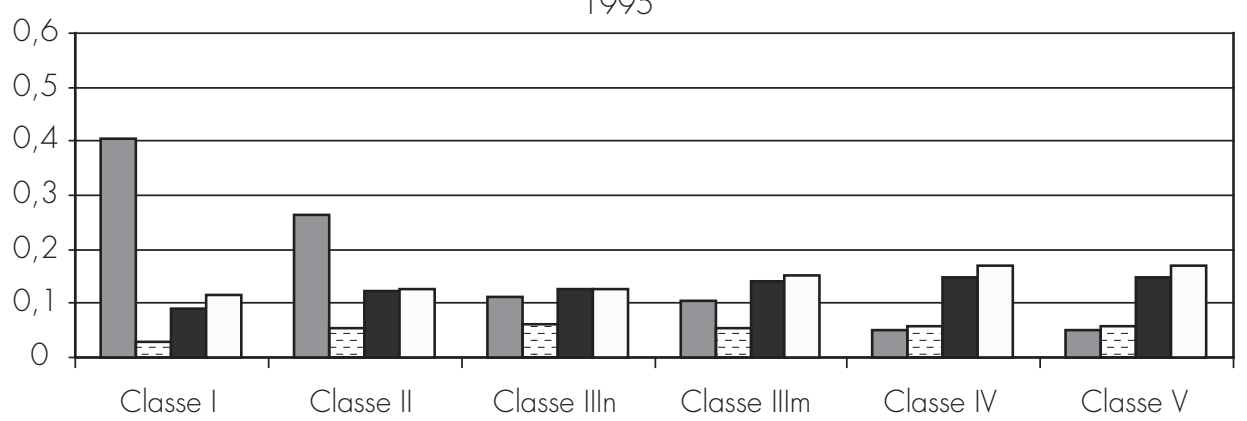

Source : SIES 1989, 1993, 1996.

(*) : Pour rappel, les classes I et II regroupent les employeurs et les cadres supérieurs, la classe IIIn les professions intermédiaires non manuelles, la classe IIIm les professions intermédiaires manuelles et les classes IV et V les professions manuelles, ordinaires et faiblement qualifiées ainsi que les personnes sans emploi. Quelques postes de ressources (épargne, autres...) ne présentaient aucune évolution notable. Ils ont donc été volontairement écartés des graphiques pour intégrer le groupe social d'origine dans l'analyse des évolutions générales mises en évidence dans la partie 3. Le poste « bourse » est lui aussi écarté. Les bourses étant sous conditions de ressources de 1988 à 1995 , l'intégration de ce poste n'aurait mis en évidence que son poids variable selon les classes.

supérieures, de 1992 à 2004, alors qu'il l'a été par sept dans les classes inférieures. Fort logiquement, on retrouve les mêmes évolutions quant à la dette moyenne déclarée par l'étudiant au moment de l'enquête ${ }^{18}$. Si

${ }^{18}$ La dette moyenne est ici minorée par le montant d'épargne déclaré au moment de l'enquête. elle a augmenté de $57 \%$ dans la catégorie supérieure de 1998 à 2004, la hausse a été, pour les catégories intermédiaires et inférieures, respectivement de $89 \%$ et $72 \%$. Ces deux franges de la population déclaraient une dette moyenne d'environ $6000 £$ (7 920 €) en 2004, un montant de moitié plus élevé que celui déclaré par le groupe supérieur. 
Graphique 3

Poids des parents, du salaire et du prêt public (Student loans company) dans le revenu moyen des étudiants à temps plein en 1998 et 2004 selon les classes sociales( ${ }^{*}$ )
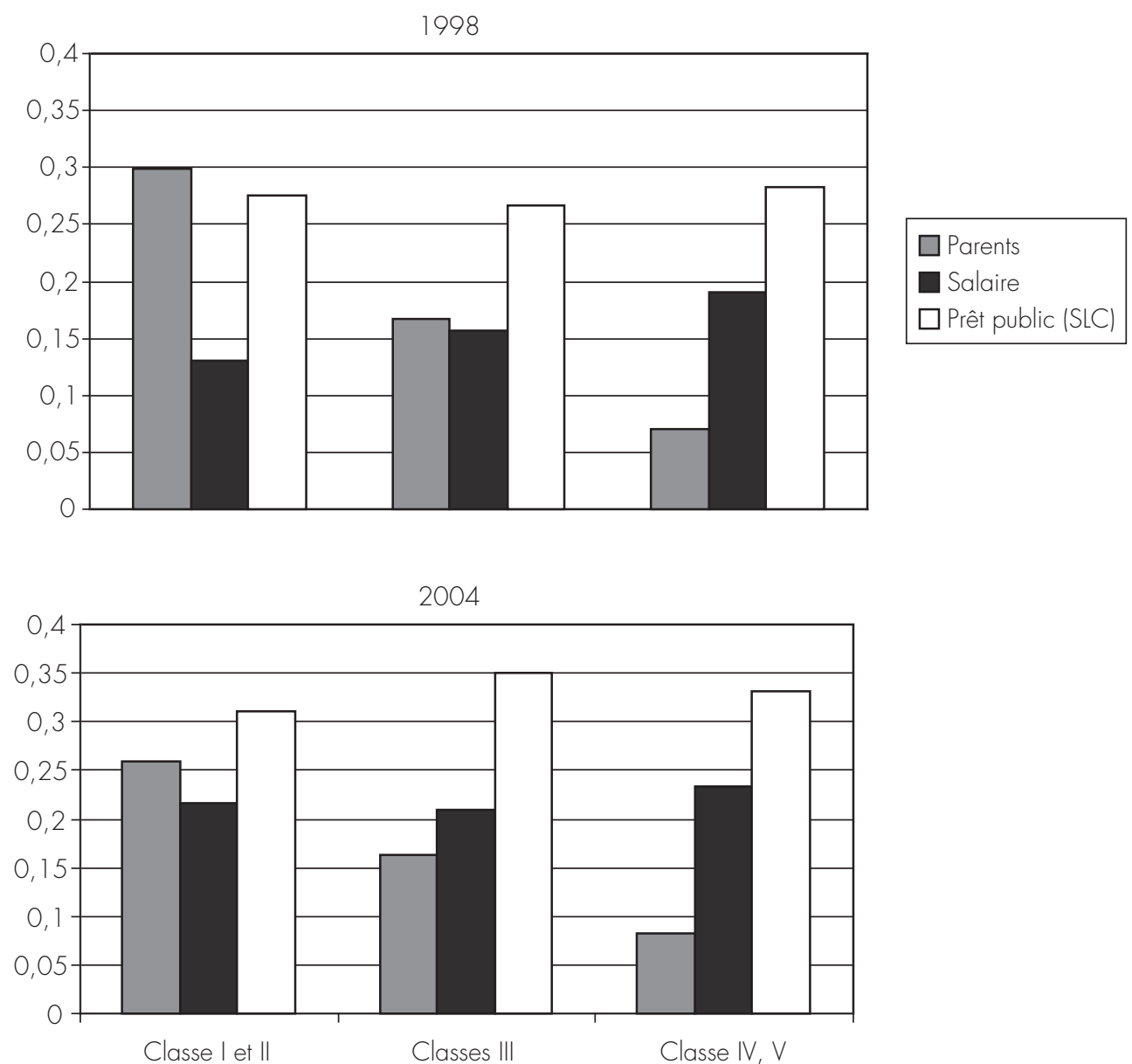

Source : SIES 2000 et 2006

(*) : Suite à un changement dans les conventions statistiques, l'épargne et le prêt commercial sont exclus de la mesure du revenu. Étant donné la suppression des bourses en 1998, leur poids se réduit considérablement dans l'ensemble des classes de 1998 à 2004.

En somme, les conservateurs ont accru, dans toutes les classes, le poids du salariat et de l'endettement. Ils ont valorisé trois nouveaux postes de financement (salaire, prêts publics, prêts commerciaux) dont le poids s'est légèrement accru dans les classes inférieures. Si les travaillistes semblent avoir égalisé le poids du salaire et des prêts publics entre les différentes classes sociales, ils ont continué de les valoriser, entraînant un déplacement plus qualitatif des inégalités de classes au niveau des heures travaillées, du salaire perçu et de la propension à recourir aux prêts commerciaux.
Les politiques publiques d'enseignement supérieur menées, depuis 1987, au Royaume-Uni, ont organisé l'expansion de l'enseignement supérieur, avec notamment le doublement des effectifs accédant à ce niveau d'éducation. Le développement des frais d'inscription et des prêts étudiants a généré un mouvement d'endettement et de mise en emploi des étudiants. Ce mouvement est fondé d'une part sur le développement et la reconnaissance des études à temps partiel 
et, d'autre part, sur le recours accru au salaire et aux prêts publics par les étudiants à temps plein. Il permet à une population de plus en plus importante d'accéder ${ }^{19}$ à un niveau d'éducation dans des conditions et des contraintes de financement de plus en plus homogènes. La composition des revenus étudiants montre toutefois des différences selon l'origine socio-économique de l'individu et selon ses modalités d'études.

19 Par « accéder », on entend ici uniquement la capacité à démarrer une année d'études dans une université. Les taux de réussite et d'abandon universitaires ne constituaient pas l'objet de cet article. Il est toutefois fort possible qu'ils aient diminué depuis le début de l'expansion. La question a été soulevée récemment par un rapport parlementaire (House of Commons, 2008) faisant état d'un maintien d'un taux d'abandon des étudiants à temps plein aux environs de 8-9 \%, et des différences existant entre établissements en la matière.
Au-delà des dispositifs publics de soutien, ces inégalités concernent surtout les étudiants à temps plein, les emplois qu'ils occupent (rémunération, temps de travail...) et leur propension à recourir aux crédits commerciaux.

Ce mouvement permet de mettre en perspective les objectifs politiques originels centrés sur la qualité des études supérieures et l'élargissement de l'accès. D'une part, il institue l'emploi et l'endettement au sein d'une population étudiante qui avait pour habitude de ne pas y recourir. D'autre part, la massification renouvelle les inégalités de classes dans le domaine de l'enseignement supérieur, l'emploi et l'endettement des étudiants à temps plein s'avérant particulièrement propices au développement de ces inégalités.

\section{Bibliographie}

Ball S., David M., Davies J. et Reay D. (2001), "Choices of degree or degrees of choice ? Class, "race" and the higher education choice process", Sociology, Vol. 35, n 4, pp. 855-874.

Callender C. (2002), "The costs of widening participation: contradictions in New Labour's student funding policies", Social Policy and Society, pp. 83-94.

Carpentier V. (2004), Historical Statistics on the Funding and Development of the UK University System, 1920-(2002), UK Data Archive, http://www. data-archive.ac.uk.

Casta A. (2007), L'action publique dans les ressources financières étudiantes en France et en Angleterre depuis les années 80 : bourses et prêts, Mémoire de Master 2, université Paris X Nanterre.

Committee of Vice-Chancellors and Principals (1989), The response of the CVCP to the Government's White Paper "Top-up Loans for Students", CVCP.

Dearing R. (sous la dir.), (1997), Higher Education in a learning society, National Committee of Inquiry Into Higher Education.
Deer C. (2004), "The expansion of higher education: economic necessity or hyper-inflation?", in Balancing the skills equation, Hayward G. et James S. (coord.), Bristol, Policy Press.

DES (1987), 1987, Higher education: meeting the challenge, DES.

DES (1988), Top-up loans for students, DES.

DES (1991), Higher education: a new framework.

DES (2003), The future of higher education, white paper on higher education.

DES (2004), Moving toward a single combined grant for higher education.

DES (2006), Widening participation in higher education.

Eymard-Duvernay F. et Favereau O. (2006), «État Social : une approche par l'Économie des Conventions », Communication au colloque État et régulation sociale : comment penser la cohérence 
de l'intervention publique ?, université Paris 1, septembre.

Galland O. et Oberti M. (1996), Les étudiants, La Découverte, coll. « Repères ».

Gary-Bobo R. et Trannoy A. (2005), « Faut-il augmenter les droits d'inscription? ", Revue française d'économie, vol. 19, janvier.

HESA (2006), Students in higher education institutions: 2004/2005.

House of Commons (2008), Staying the course: the retention of students on higher education courses, 4 février.

IREDU (1977), L'aide aux étudiants en France : faits et critique, CNRS.

Maurin E. (2007), La nouvelle question scolaire, Seuil.

NUS (2007), The great higher education funding debate.

Pennell H. et West A. (2005), "The impact of increased fees on participation in higher education in England", Higher Education Quarterly, Vol. 59, n² april, pp. 127-137.
Simmel G. (1987), Philosophie de l'argent, PUF.

Simmel G. (1998), Les pauvres, PUF.

SLC (1998), Statistics of Education : student support, England and Wales, 1996/97.

SLC, (2007), Student loan for higher education in England, financial year 2006/07 (provisional), First Release.

Stanton G. (2008), “Access to higher education in England: its position within the wider educational ecology", in Unfinished business in widening participation, Copland G., Sachdev D. et Flint C. (ed.), LSN.

TUC (2008), All work and low pay.

UUK (2006), Part-time students and part-time study in higher education in the UK : a survey of students' attitudes and experiences of part-time study and its costs 2005/06, UUK, strand 3.

Vinokur A. (2007), « Study Now, Pay Later. Endettement étudiant et restructuration de l'enseignement supérieur », in Vinokur A. (coord.) Pouvoirs et financement en éducation, Qui paye décide ?, Paris, L'Harmattan, coll. «Éducations et sociétés ».

\section{Résumé}

\section{La nouvelle condition des étudiants anglais : entre endettement et emploi \\ Aurélien Casta}

Les politiques publiques menées depuis 1987, au Royaume-Uni, ont organisé une expansion de l'enseignement supérieur, imposant notamment un doublement des effectifs accédant à ce niveau d'éducation. L'analyse des enquêtes sur les revenus étudiants, réalisées depuis 1990, met en lumière un développement de l'emploi et de l'endettement des étudiants qui notamment révèle deux des soubassements de cette expansion. D'une part, les politiques publiques conduites dans le cadre de l'expansion instituent l'emploi au sein d'une population qui avait l'habitude de ne pas y recourir. D'autre part, ce phénomène, tout comme l'endettement, touche plus particulièrement les étudiants à temps plein issus des classes populaires.

Mots clés :

Royaume Uni, étudiant, accès à l'enseignement supérieur, travail étudiant, financement de l'enseignement supérieur, classe ouvrière, politique publique

Journal of Economic Literature: I 23 